\title{
Painful and painless ophthalmoplegia with cavernous sinus pseudotumour
}

Great Ormond Street Hospital for Children NHS Trust, London: Department of Paediatric Neurology V Ganesan J-P Lin F J Kirkham

R A H Surtees

Department of

Radiology

W K Chong

Correspondence to: Dr V Ganesan, The Wolfson Centre, Mecklenburgh Square, London WC1N 2AP.

Accepted 10 May 1996

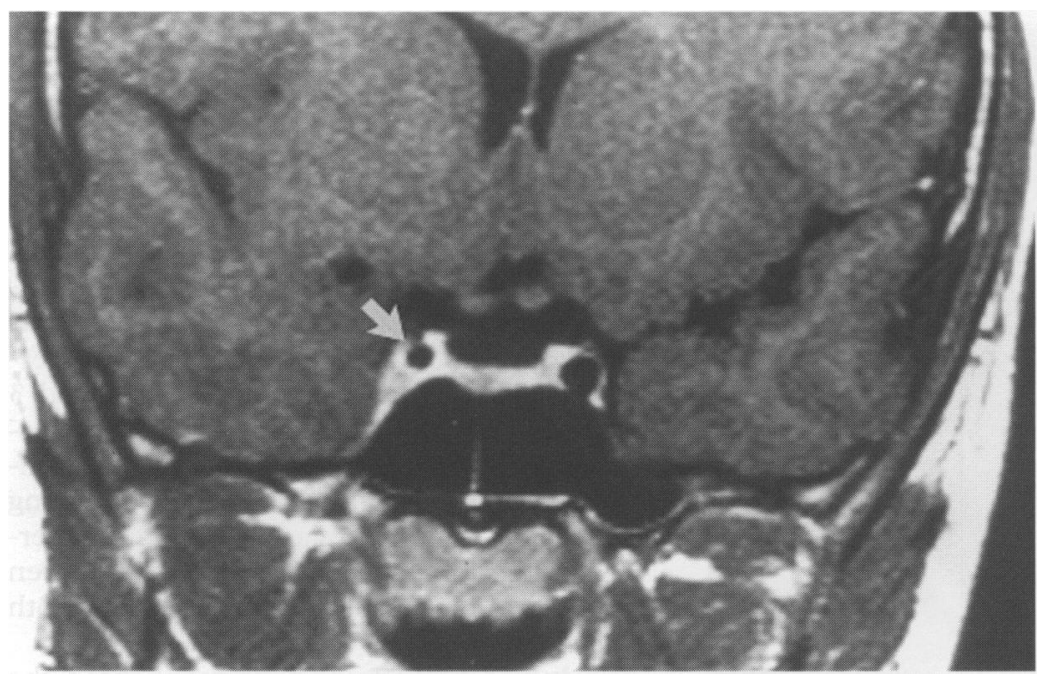

Figure $1 T 2$ weighted magnetic resonance image of case 1 (coronal section). The image shows concentric narrowing of the intracavernous portion of the right internal carotid artery due to external compression by enhancing tissue. migraine or post-infectious isolated cranial neuropathy. However, our cases show that cavernous sinus pseudotumour can present as isolated ophthalmoplegia, either with or without pain. Since these children may present to a general practitioner, paediatrician, paediatric neurologist, or ophthalmologist, we hope to use these cases to increase diagnostic awareness of this treatable condition.

\section{Case 1}

A 13 year old boy presented with a 10 day history of diplopia and difficulty moving his right eye. Despite intensive inquiry, he denied any headache or orbital pain. He had no other relevant medical history and was otherwise well. On examination he had a complete right VIth nerve palsy. The eye was not inflamed or proptosed; visual acuity and colour vision were normal. There were no other neurological signs and general examination, including blood pressure, was normal.

A magnetic resonance imaging (MRI) scan of the brain with gadolinium showed enhancing tissue in the right cavernous sinus with concentric narrowing of the intracavernous portion of the right internal carotid artery (fig 1); the appearances were consistent with inflammatory pseudotumour.

There was evidence of a non-specific autoimmune diathesis since the gastric parietal cell antibody titre was greatly raised initially $(1: 1280)$ and fell in the convalescent period. All other investigations, including the erythrocyte sedimentation rate (ESR), were normal.

He was initially managed conservatively. Six weeks later there was no improvement in either the clinical or MRI findings. Due to concern about a permanent divergent squint, he was offered botulinum toxin injection into the right medial rectus in order to prevent permanent strabismus due to the unopposed activity of this muscle. This was declined by the patient; instead, a trial of prednisolone, $2 \mathrm{mg} / \mathrm{kg} / \mathrm{day}$, was begun. There was a gradual but complete resolution of the VIth nerve palsy over the following month. Steroids were withdrawn successfully with no recurrence of his symptoms.

Case 2

An 11 year old boy of Afro-Caribbean extraction developed left fronto-orbital headache and vomiting. Twenty four hours later he was unable to open his left eye which was described as being deviated downwards and towards the left. Although the headache and nausea resolved, the ophthalmoplegia was persistent. An initial diagnosis of ophthalmoplegic 
migraine was made. Two weeks later, the headache and nausea recurred for a day. A week later, these symptoms recurred again and a computed tomography (CT) scan of the brain was carried out. This was initially considered to be normal and he was referred for a neurosurgical opinion, and in particular for exclusion of a carotid artery aneurysm. There was no other relevant history and no personal or family history of migraine or sickle cell disease.

On examination he was afebrile and had a left IIIrd nerve palsy with pupillary sparing. The eye was not inflamed, tender, or swollen and there was no proptosis. Visual acuity and colour vision were normal. Detailed cranial nerve examination, including examination of facial sensation, revealed no other deficits and neurological and systemic examination were otherwise normal.

Magnetic resonance imaging of the brain showed abnormal enhancing tissue extending from the left cavernous sinus posteriorly along the free edge of the tentorium and also towards the orbital apex (fig 2). On review, this abnormality was apparent on the initial CT scan.

Extensive investigation revealed no underlying inflammatory or autoimmune process.

Prednisolone was started at a dose of 2 $\mathrm{mg} / \mathrm{kg} /$ day. Following this, there was no recurrence of the headache or nausea. After five weeks of steroids, there was partial resolution of the ophthalmoplegia with improvement of the ptosis; he was able adduct the left eye to the

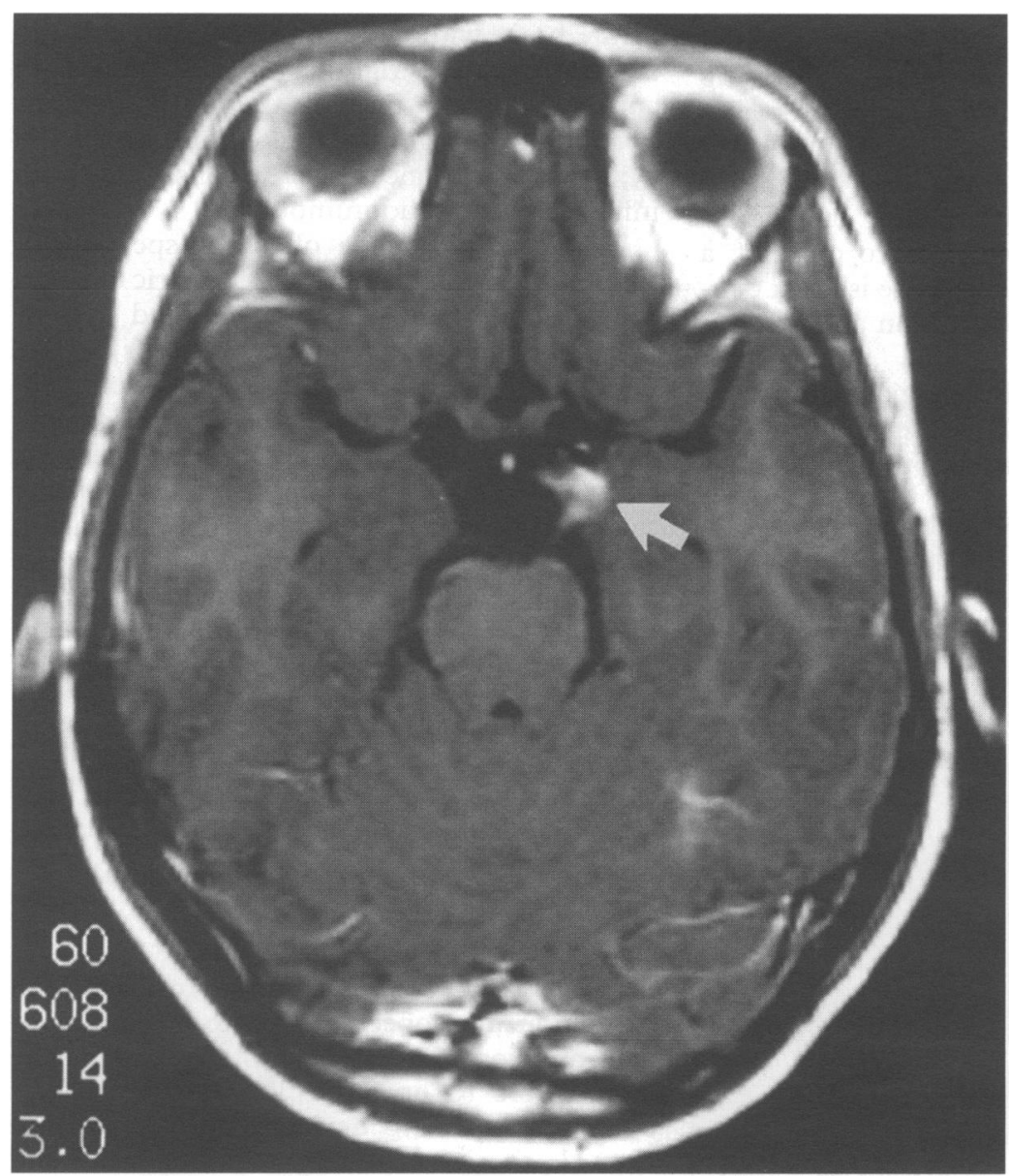

Figure 2 T2 weighted magnetic resonance image of case 2 (axial section) showing enhancing tissue in the left parasellar region. midline but not beyond, and his diplopia had resolved. Six months after his initial presentation, the ophthalmoplegia had fully resolved.

\section{Discussion}

The term 'pseudotumour' is used in this context to describe a mass of inflammatory tissue of unknown origin. Tolosa's original patient had exploratory surgery for painful ophthalmoplegia and died postoperatively. Granulomatous tissue was discovered in the cavernous sinus. Histological analysis of the tissue revealed non-specific features of inflammation. ${ }^{1}$ Hunt et al subsequently published a series of six cases and laid down clear diagnostic criteria. ${ }^{2}$ This series also referred specifically to the presence of pseudotumour within the cavernous sinus. Subsequently, it was observed that this condition responded well to steroids. $^{3}$

The eponymous description of Tolosa-Hunt syndrome is used in published reports to refer not only to cavernous sinus pseudotumour but also to orbital and retro-orbital pseudotumour. This has clouded recognition of possible pathology within the cavernous sinus since this can present-unlike orbital pseudotumourwithout signs of orbital inflammation, chemosis, or proptosis. We are aware of no previous report of pseudotumour in the cavernous sinus causing painless ophthalmoplegia, as in our first patient, and the spectrum of symptoms and clinical presentations may be wider than was previously recognised.

Magnetic resonance imaging has enabled detailed imaging of the cavernous sinus and has enhanced diagnosis of idiopathic inflammatory pseudotumour. ${ }^{4}$ The availability of this imaging mode has superseded Hunt's original caveat that this diagnosis could only be made if surgical exploration and angiography were negative. ${ }^{2}$ However, this usually means that histological confirmation of the diagnosis is not available and it is important to recognise that the differential diagnosis of the radiological appearances is large. A similar clinical picture can also be produced by other conditions, including neoplastic and vascular lesions, notably carotid aneurysms.

Although granulomatous diseases such as sarcoidosis or tuberculosis can present with involvement of the cavernous sinus, this is rare. Indices of acute inflammation or infection are usually normal or non-specifically abnormal, as in our patients.

Gordon, ${ }^{5}$ in his review of the differential diagnosis of painful and painless ophthalmoplegia in childhood, quotes the cases of TolosaHunt syndrome in childhood reported by Terence and Samaha. ${ }^{6}$ The children were aged 3 and 8 years old at onset and had either periorbital or retro-orbital pain. Both were extensively investigated, although no neuroimaging was available. All investigations, including cerebral angiography, were normal. Both children responded to prednisolone, although they both relapsed later.

Kandt and Goldstein ${ }^{7}$ have highlighted the difficulty of distinguishing steroid responsive ophthalmoplegia from ophthalmoplegic mi- 
graine and recommend a trial of steroids in cases where the ophthalmoplegia persists. Kuzemko and Young $^{8}$ reported a case of ophthalmoplegic migraine with proptosis and blurring of the optic discs. Contrast angiography revealed narrowing of the internal carotid artery; this was interpreted to represent vasospasm although, as illustrated in fig 1, external compression from intracavernous pseudotumour can cause this appearance. It seems reasonable to speculate that these cases represented cavernous sinus pseudotumour and that clarification of the pathogenesis of ophthalmoplegia in childhood is only now possible with high resolution neuroimaging.

Despite steroid responsiveness, it is vital that clinicians remain vigilant and that the patient's clinical state and the radiological features are monitored closely as symptoms due to other conditions, including meningiomas, lymphoma, and aneurysms, ${ }^{9}$ can show an initial response to steroids. Although short term symptomatic treatments, such as the botulinum toxin injection offered to case 1 , may be useful in the management of strabismus, diagnosis and specific treatment of the underlying cause is definitive and should remain a priority. In the case of pseudotumour, relapse can occur and may involve the contralateral side, and the patient can be left with residual deficits. Despite the relative sensitivity of magnetic resonance imaging, carotid angiography or surgical exploration and biopsy may be necessary eventually to exclude more sinister pathology and to obtain a tissue diagnosis. The diagnosis of persistent migrainous ophthalmoplegia should only be accepted after definitive exclusion of pseudotumour.

1 Tolosa E. Periarteritic lesions of the carotid siphon with the clinical features of a carotid infraclinoidal aneurysm. $f$ Neurol, Neurosurg Psychiatry 1954;17:300-2.

2 Hunt WE, Meagher JN, LeFever HE, Zeman W. Painful ophthalmoplegia. Its relation to indolent inflammation of

the cavernous sinus. Neurology 1961;11:56-62.
3 Hunt WE. Tolosa-Hunt syndrome: one cause of painful Hunt WE. Tolosa-Hunt syndrome: one cause
ophthalmoplegia. J Neurosurg 1976;44:544-9.

4 Goto Y, Hosokawa S, Goto I, Hirakata R, Hasuo K. Abnormality in the cavernous sinus in three patients with TolosaHunt syndrome: MRI and CT findings. $f$ Neurol, Neurosurg Psychiatry 1990;53:231-4.

5 Gordon N. Ophthalmoplegia in childhood. Dev Med Child Neurol 1994;36:370-4.

6 Terence CF, Samaha FJ. The Tolosa-Hunt syndrome (painful ophthalmoplegia) in children. Dev Med Child Neurol 1973;15:506-9.

7 Kandt RS, Goldstein W. Steroid-responsive ophthalmoplegia in a child. Diagnostic considerations. Arch Neurol 1985; 42:589-91.

8 Kuzemko JA, Young W. Ophthalmoplegic migraine: a case report. Dev Med Child Neurol 1967;9:427-9.

9 Wroe SJ, Thompson AJ, McDonald WI. Painful intraorbital meningiomas. $\mathcal{F}$ Neurol Neurosurg Psychiatry 1991;54:1009- 